\title{
The semantics of plane-mirror inversion
}

Those who ask why mirrors "invert right and left", but not "top" and "bottom", must not look for help in physics textbooks, but in dictionaries that define the use of words and in an understanding of reality.

IT is, of course, a commonplace that people will say that an image in a plane mirror is inverted in the sense that 'right' is replaced by 'left'. You only have to look, and you will see! Hold up your right hand, palm forward, in front of a plane mirror, and you will see what looks like a left hand facing back at it. Moreover, the image is clearly not just a flat two-dimensional cutout; if you keep the hand fixed, but move your head, the mirror will reflect light from a slightly different subsurface of your hand, and so the image will seem itself to be three-dimensional.

If all this is accepted, then an uncomfortable question usually arises: if reflection in a plane mirror inverts right and left, why does it not also invert top and bottom? Often one comes across explanations based on the assertion that the horizontal plane is distinguished from the vertical in that human beings have two eyes which, between them, define a unique horizontal plane, but that there is no means by which a unique vertical plane is defined by the physiology of the onlooker. There are all kinds of philosophical objections to arguments of that kind, but the most telling way of turning them is the simple observation that even a one-eyed man will see the reflection of his right hand in a plane mirror as what seems to be a left hand. Another is to suppose that the observer reclines horizontally in front of a plane mirror; again there is no inversion of top and bottom, but again a right hand is reflected in an image looking like a left hand.

So what is the explanation? E. C. Horsfield from the University of Durban has now persuaded the European Journal of Physics to let him publish a solemn discussion of the issue $(12,207 ; 1991)$ which appropriately begins with quotations from well-known undergraduate texts asserting that "images in a plane mirror have right and left interchanged". They are all, of course, "fallacious".

Horsfield's view is that the underlying error in these statements is that their authors confuse "physics and psychology" - the reflection of light in mirrors and what observers construe of the images they see. And, correctly, he points out that the only possible effect of a plane mirror that may be taken as inversion of any kind is that in which an array of points on a straight line perpendicular to the surface of the mirror will appear, in reflection, to be inverted. There is no lateral displace- ment of any kind, such as would be needed to bring about left-right or even top-bottom inversion, but only inversion in a direction perpendicular to the plane.

That point is easily verified. If $\mathbf{X}$ is a vector in front of and anchored into the surface of the mirror, and $\mathbf{n}$ a unit vector perpendicular to the mirror anchored at the same point, the component of $\mathbf{X}$ perpendicular to the mirror is $(\mathbf{X} \cdot \mathbf{n}) \mathbf{n}$ and the component in the surface of the mirror is simply $\mathbf{X}-(\mathbf{X} \cdot \mathbf{n}) \mathbf{n}$. The effect of reflection is simply to replace $\mathbf{n}$ with $-\mathbf{n}$, so that the reflection of the vector $\mathbf{X}$ is simply $\mathbf{X}-2(\mathbf{X} \cdot \mathbf{n}) \mathbf{n}$. All that means is that the image of a point a certain distance in front of the mirror is displaced by twice that distance towards the other side of the surface, and that there are no lateral displacements.

How, then, does the illusion of leftright displacement arise? Horsfield goes on to argue that, whatever the mechanism, the illusion is incomplete. A person with hair parted on the left who looks into a mirror will see an image of what appears to be a person with hair parted on the lefthand side. But if the image is to be taken as that of the face of a real person, the parting of the hair will be on that person's right. So the image does not correspond exactly to the appearence of the person in front of the mirror. And the lateral inversion occurs only in "the observer's mind".

Horsfield attributes the root of the trouble to people's common determination to personify the images they see as reflections in mirrors. By way of antidote, it could be worthwhile for all of us to compare our reflections in a mirror with a photograph taken by a camera, when the two images would again suffer left-right inversion. But the only long-term remedy is to believe the truth - that all images are virtual images, without reality, just as are the virtual images supposed in the elementary optics textbooks to be formed in front of (not behind) convex spherical mirrors.

Astronomers and those who read their journals are well used to the difficulty: photographs of the sky habitually have 'East' on the left and 'West' on the right, yet nobody seems to turn a hair. The verbal explanation is simple. The mirror image of the sky is simply the projection onto a mirror of what seems to be a surface full of steller objects. One's view of the image is then what one would obtain if it were possible to position oneself behind the stellar surface to observe the arrangement of the objects. It is as if the object in view is flattened onto the mirror, and one is enabled to look at it from behind. This is also what happens with the image of one's face in a plane mirror; the mirror captures a front view of one's face and also enables one to look at it from behind. The trick amounts to nothing more than the inversion of the perpendicular components of all vectors in the mirror surface.

Grand philosophical questions naturally arise, but should be dismissed. There is, for example, the issue of 'reality', much discussed by people such as Hume and Kant. Is the image in a mirror real? Clearly it is not. The most that can be said is that an observer can reconstruct the supposedly real object that generates the image once the rules are known - that the left seems to appear on the right, for example.

But even that does not put an end to philisophical objections. Who, after all, can know what exactly happens within our heads in the direct perception by the eyes of objects placed in front of them? However perception works, may it not be likened to a system of mirrors that leads, willy-nilly, to the formation of an image by means that are in principle no different from the formation of an 'inverted' image in a plane mirror? And while all the members of a group standing round, say, a table will be tempted to vote for the proposition that "That is a table", there is no objective way of demonstrating that all of them are seeing the same thing.

Historically, arguments like these prompted Ernst Mach's positivist view of reality - that the only attributes of a physical object with significance are its measurable attributes. But even that is only half the battle. Have not generations of young people been drilled at school to measure the 'position' of a mirror image by sticking pins in an optical bench, looking for the position at which the image appears not to move? The truth is that these experiments do not measure an attribute of the image, but rather identify a fixed point from which all rays reflected in a mirror from a common origin appear to diverge. The trouble is simply semantic that of pretending that the image is an object. The remedy is not the obvious abolition of 'image', for the word is altogether too useful for that. The best course is simply to remember that all images are unreal.

John Maddox 